\title{
Edge Caching Resource Allocation in Fog Radio Access Networks: An Incentive Mechanism Based Approach
}

\author{
Hui $\mathrm{Ge}^{1,2,3}$, Yanxiang Jiang ${ }^{1,2,3, *}$, Mehdi Bennis ${ }^{4}$, Fu-Chun Zheng ${ }^{1,5}$, and Xiaohu You ${ }^{1}$ \\ ${ }^{1}$ National Mobile Communications Research Laboratory, Southeast University, Nanjing 210096, China \\ ${ }^{2}$ State Key Laboratory of Integrated Services Networks, Xidian University, Xi' an 710071, China \\ ${ }^{3}$ Key Laboratory of Wireless Sensor Network \& Communication, Shanghai Institute of Microsystem and \\ Information Technology, Chinese Academy of Sciences, 865 Changning Road, Shanghai 200050, China \\ ${ }^{4}$ Centre for Wireless Communications, University of Oulu, Oulu 90014, Finland \\ ${ }^{5}$ School of Electronic and Information Engineering, Harbin Institute of Technology, Shenzhen 518055, China \\ *E-mail: yxjiang@seu.edu.cn
}

\begin{abstract}
In this paper, an edge caching resource allocation problem in fog radio access networks (F-RANs) is investigated. To motivate content providers (CPs) to participate in this resource allocation procedure, we introduce an incentive mechanism. By treating fog access points (F-APs) as a specific type of edge caching resource, the cloud server sets non-uniform prices of F-APs and leases them to the CPs, while the CPs cache the most popular contents in the storage of F-APs and get rewarded by the raised content hit rate. We formulate the interaction between the cloud server and the CPs as a Stackelberg game and solve the corresponding optimization problems to achieve Nash equilibrium (NE). In particular, by exploiting the multiplier penalty function method, we transform the constrained optimization problem for the cloud server into an equivalent non-constrained optimization problem. Then, we propose an edge caching resource pricing algorithm to solve the non-constrained optimization problem by applying the simplex search method. We also theoretically prove the existence and uniqueness of the NE. Simulation results show the rapid convergence of the proposed algorithm and the superiority performance in improving content hit rate.
\end{abstract}

Index Terms-F-RANs, edge caching, resource allocation, incentive mechanism, Stackelberg game.

\section{INTRODUCTION}

Driven by the dramatic growth of intelligent devices and mobile applications, wireless networks have been suffering an unprecedented data traffic pressure in recent years. Meanwhile, numerous repetitive downloads and redundant transmissions occur when users request the same contents. Nowadays, fog radio access networks (F-RANs), which can effectively accommodate the data traffic pressure by placing popular contents close to the users, have attracted more and more attention from researchers and engineers [1]-[3].

In F-RANs, fog access points (F-APs) managed by the cloud server are able to store contents in their storage during off-peak time. When user equipments (UEs) request these contents, the F-APs having cached the corresponding contents can provide service directly. Accordingly, the data traffic pressure can be reduced while the quality of service can also be improved. However, the resource, especially the edge caching resource, is limited. Therefore, how to optimally allocate resource becomes a big challenge.

Recently, large amounts of researchers have paid attention to resource allocation problem from different aspects. The authors in [4] studied a joint resource allocation and content caching problem which targeted at minimizing the maximum content request rejection rate. In [5], a problem of joint caching, channel assignment, and interference management was formulated in coordinated small-cell cellular networks to maximize the system throughput. In addition, it becomes an interesting topic to solve the resource allocation problem from economic perspective. Game theory has been widely utilized to solve this problem [6]. In [7], a caching system consisting of one privately-owned small-cell base station (SBS) and multiple content providers (CPs) was studied, where the CPs leveraged the storage capacity of the SBS to efficiently provide content delivery service to UEs. A Stackelberg game was formulated for the interaction between the SBS and the CPs. Nevertheless, the authors do not take the spatial distribution of SBSs into consideration. Moreover, the proposed uniform pricing scheme ignores the diversity of the CPs. In [8], a pricebased resource allocation policy was proposed to reduce the transmission latency and mitigate the redundant transmission in a commercialized caching system. The authors considered the spatial distribution of SBSs and proposed a non-uniform resource pricing scheme. However, if the average back-haul cost was not equal to the local downloading surcharge, the objective function then could not be simplified and the scheme would be ineffective.

Motivated by the aforementioned discussions, we propose an edge caching resource allocation strategy in F-RANs. By regarding F-APs as a type of edge caching resource, the cloud server is willing to profit by leasing F-APs to the CPs. However, renting F-APs will cause cost while the CPs have no incentive to rent. Therefore, we propose an incentive mechanism to motivate the CPs to participate in this resource allocation procedure. In our proposed strategy, the CPs can benefit from the content hit rate by caching their most popular contents in the leasing F-APs, while the cloud server can also make profit by charging the CPs with non-uniform prices for F-APs. The interaction between the cloud server and the CPs is modeled as a Stackelberg game. Meanwhile, the CPs will compete with each other due to the limited edge caching resource. Therefore, we formulate the competition among the CPs as a non-cooperative sub-game. To achieve 
the NE of the proposed game, we eliminate the constrains by exploiting the multiplier penalty function method, and propose an edge caching resource pricing algorithm with the simplex search method. In addition, we also prove the existence and uniqueness of the NE.

The rest of this paper is organized as follows. In Section II, the system model is presented. In Section III, the profit functions are established and the Stackelberg game is also formulated. We propose the optimal edge caching resource allocation strategy by solving the optimization problems in Section IV. Simulation results are shown in Section V. Conclusions are drawn in Section VI.

\section{SYSTEM MODEL}

We consider an edge caching resource allocation problem in the F-RAN including one cloud server and multiple F-APs. As illustrated in Fig. 1, there are $N$ CPs, which are denoted by $\mathcal{N}=\{1,2, \ldots, n, \ldots, N\}$, willing to cache their contents in the storage of F-APs.

The contents owned by the CPs have different popularity distributions. There are totally $C_{n}$ contents stored in CP $n$. The UEs make independent requests of the $f$ th content owned by CP $n$ with the probability of $p_{n, f}$. Generally, the popularity can be modeled by the Zipf distribution [9] and $p_{n, f}$ can be expressed as follows:

$$
p_{n, f}=\frac{1 / f^{\beta_{n}}}{\sum_{f=1}^{C_{n}} 1 / f^{\beta_{n}}}, \forall n \in \mathcal{N}, \forall f \in C_{n},
$$

where $\beta_{n}$ denotes the steepness of the popularity distribution in CP $n$ with a positive value.

In the F-RAN, F-APs are spatially distributed as a homogeneous Poisson point process (HPPP) with intensity $\lambda$ [10], where $\lambda$ denotes the number of F-APs per unit area. Furthermore, each F-AP with the same storage capacity can cache at most $Q_{n}$ contents for $\mathrm{CP} n$. Let $\tau_{n}$ represent the fraction of the total F-APs in this scenario leased by $\mathrm{CP} n$. Meanwhile, the rented F-APs of CP $n$ are selected with equal probability. Therefore, the distribution of these rented F-APs can be modeled as thinned HPPP with intensity $\tau_{n} \lambda$. Assume that each F-AP can provide service for UEs within range $r$. Let $H_{n}$ denote the probability for UEs covered by any F-AP

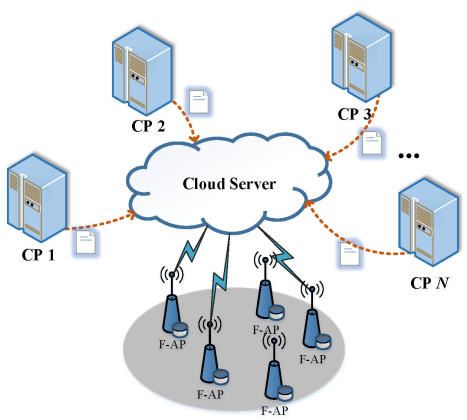

Fig. 1. An illustration of the system model. leased by $\mathrm{CP} n$. Then, $H_{n}$ can be expressed as follows [11]:

$$
H_{n}=1-\exp \left(-\pi \tau_{n} \lambda r^{2}\right) .
$$

If $\mathrm{CP} n$ has cached the $f$ th content into its renting F-APs, the hit rate of the $f$ th content could be expressed as follows:

$$
H_{n, f}=p_{n, f}\left[1-\exp \left(-\pi \tau_{n} \lambda r^{2}\right)\right] .
$$

\section{PRoblem Formulation}

In this section, we first model the profits of the cloud server and the CPs, respectively. Then, we formulate the edge caching resource allocation problem as a Stackelberg game. To motivate the CPs to participate in this resource allocation procedure, we introduce an incentive mechanism. The incentive mechanism is controlled by the charge price determined by the cloud server. In other word, the cloud server can attract the CPs to lease F-APs by setting proper prices. Moreover, the NE of the proposed Stackelberg game is also investigated.

\section{A. The Profits of the Cloud Server and the CPs}

For the cloud server, the profit comes from leasing F-APs to the CPs. Let $s_{n}$ denote the price charged to CP $n$ for renting all F-APs in the scenario, and $s \triangleq\left[s_{1}, s_{2}, \ldots, s_{n}, \ldots, s_{N}\right]^{T}$. Define $\tau_{n}$ the fraction of the F-APs rented by CP $n$, and $\tau \triangleq$ $\left[\tau_{1}, \tau_{2}, \ldots, \tau_{n}, \ldots, \tau_{N}\right]^{T}$. Then, the profit function of the cloud server can be expressed as follows:

$$
P_{c}(\boldsymbol{s}, \boldsymbol{\tau})=\sum_{n=1}^{N} \tau_{n} s_{n} .
$$

For each CP, the profit consists of two parts: (1) the gain brought by the content hit rate; (2) the cost of renting F-APs from the cloud server. To achieve the maximal content hit rate, CP $n$ will cache the most popular contents in the leasing FAPs. Therefore, we can define the profit function for $\mathrm{CP} n$ as follows:

$$
P_{n}\left(s_{n}, \tau_{n}\right)=\sum_{f=1}^{Q_{n}} H_{n, f}-s_{n} \tau_{n}
$$

\section{B. Stackelberg Game Formulation}

Generally, Stackelberg game is a strategic game that consists of a leader and several followers competing with each other for certain resources [12]. The leader moves first and the followers move subsequently. In our Stackelberg game, the cloud server that manages the F-APs is the leader and the CPs willing to lease F-APs are the followers. Besides, due to the limited edge caching resource, it is easy to see that the CPs strictly compete with each other in a non-cooperative fashion. Therefore, the sub-game of the proposed Stackelberg game is modeled as a non-cooperative game. The cloud server first determines the leasing prices $s$ of F-APs for the CPs. According to the proposed prices, the CPs determine the proper fractions $\tau$ that they tend to rent.

1) Optimization Problem of the Cloud Server: The objective of the cloud sever is to maximize its profit formulated in 
(4). Note that for CP $n$, the leasing fraction $\tau_{n}$ is a function of the price $s_{n}$ in the Stackelberg game formulation. In other words, the fraction of F-APs that each CP wants to rent depends on the price charged by the cloud server. Consequently, the cloud server needs to find the optimal pricing strategy to maximize its profit. The optimization problem of the cloud server can be formulated as follows:

$$
\begin{array}{ll} 
& \max _{\boldsymbol{s}} P_{c}(\boldsymbol{s}, \boldsymbol{\tau}), \\
\text { s.t. } & s_{n} \geq 0, \forall n \in \mathcal{N} .
\end{array}
$$

2) Optimization Problem of the CPs: For $\mathrm{CP} n$, once the price of F-APs is determined, its profit all depends on the leasing fraction $\tau_{n}$. If CP $n$ rents more F-APs from the cloud server, it can cache contents at more F-APs which will increase the content hit rate and hence bring it more gain. However, the cost increases as the leasing fraction $\tau_{n}$. Therefore, $\tau_{n}$ has to be optimized for maximizing the profit of $\mathrm{CP} n$. The optimization problem can be expressed as follows:

$$
\begin{array}{ll} 
& \max _{\tau_{n}} P_{n}\left(s_{n}, \tau_{n}\right), \\
\text { s.t. } & 0 \leq \tau_{n} \leq 1, \forall n \in \mathcal{N}, \\
& \sum_{n=1}^{N} \tau_{n} \leq 1 .
\end{array}
$$

The optimization problems in (6) and (7) lead to a Stackelberg game. The objective of this game is to find the NE point where neither the cloud server nor the CPs have incentives to deviate [8], [13].

\section{Nash Equilibrium}

For the proposed game, the NE is defined as follows.

Definition 1: Let $s^{*}$ and $\tau^{*}$ denote the two solutions of the optimization problems in (6) and (7), respectively. Define $s^{*} \triangleq$ $\left[s_{1}^{*}, s_{2}^{*}, \ldots, s_{n}^{*}, \ldots, s_{N}^{*}\right]^{T}, \tau^{*} \triangleq\left[\tau_{1}^{*}, \tau_{2}^{*}, \ldots, \tau_{n}^{*}, \ldots, \tau_{N}^{*}\right]^{T}$. Then, $\left(\boldsymbol{s}^{*}, \boldsymbol{\tau}^{*}\right)$ is an NE point for the proposed Stackelberg game if the following conditions are satisfied:

$$
\begin{aligned}
& P_{c}\left(s^{*}, \tau^{*}\right) \geq P_{c}\left(s, \tau^{*}\right), \\
& P_{n}\left(s_{n}^{*}, \tau_{n}^{*}\right) \geq P_{n}\left(s_{n}^{*}, \tau_{n}\right), \forall n \in \mathcal{N} .
\end{aligned}
$$

Note that the proposed game is a two-layer game. We intend to exploit the backward induction method to achieve the NE [12]. Accordingly, the optimal solution of the sub-game should be obtained at first. The sub-game formulating the competition among the followers is a non-cooperative game, and its NE is defined as the operating point at which no players can improve utility by changing its strategy unilaterally [8]. It means that we have to solve the optimization problem in (7) to obtain the best responses of the CPs firstly. Since there is only one leader in the proposed Stackelberg game, the best response of the cloud server can be obtained sequentially by solving the optimization problem in (6).

\section{Optimal Resource Allocation Strategy}

In this section, we will solve the optimization problem in (7) to obtain the optimal leasing strategies of the CPs at first.
Then, the optimal pricing strategy of the cloud server will be obtained by solving the optimization problem in (6).

\section{A. Optimal Leasing Strategy}

It is obvious that the profit function in (7) is concave over $\tau_{n}$. Then, the optimal solution can be obtained by solving the Karush-Kuhn-Tucker (KKT) conditions [14], [15]. Correspondingly, we have the following theorem.

Theorem 1: Given a fixed price vector $s$, the optimal solution of the optimization problem in (7) can be expressed as follows:

$$
\tau_{n}^{*}=\left[\ln \left(\frac{F_{n}}{s_{n}}\right) / \pi \lambda r^{2}\right]^{+},
$$

where $F_{n}=\sum_{f=1}^{Q_{n}}\left(p_{n, f} \pi \lambda r^{2}\right),(x)^{+} \triangleq \max (0, x)$.

Proof: The second derivative of $P_{n}$ with respect to $\tau_{n}$ can be expressed as follows:

$$
\frac{\partial^{2} P_{n}}{\left(\partial \tau_{n}\right)^{2}}=-F_{n} \pi \lambda r^{2} \exp \left(-\pi \tau_{n} \lambda r^{2}\right)<0 .
$$

The profit function $P_{n}$ of $\mathrm{CP} n$ can be readily proved to be strictly concave. By solving $\frac{\partial P_{n}}{\partial \tau_{n}}=0$ under the constraint $\tau_{n} \geq 0$, the optimal leasing strategy $\tau_{n}^{*}$ of $\mathrm{CP} n$ can be obtained.

We can see from Theorem 1 that if the price $s_{n}$ is set too high, i.e., $s_{n} \geq F_{n}$, CP $n$ will opt out for renting any F-APs from the cloud server. In other words, $\mathrm{CP} n$ will not participate in the game due to the high price. On the other hand, if the price $s_{n}$ is lower than $F_{n} e^{-\pi \tau_{n} \lambda r^{2}}$, the competition among the $\mathrm{CPs}$ would be more intense since each $\mathrm{CP}$ is willing to rent F-APs as much as possible.

\section{B. Optimal Pricing Strategy}

Substituting the optimal solution (9) into (4) and after some mathematical manipulations, we can express the profit function for the cloud server as follows:

$$
P_{c}(s)=\sum_{n=1}^{N} \frac{1}{\pi \lambda r^{2}} \xi_{n}\left(s_{n} \ln F_{n}-s_{n} \ln s_{n}\right),
$$

where $\xi_{n}$ is the indicator function with $\xi_{n}=1$ if $s_{n} \leq F_{n}$ and $\xi_{n}=0$ otherwise. We can observe that the function in (11) is non-convex due to $\xi_{n}$. However, with a given indicator vector $\xi \triangleq\left[\xi_{1}, \xi_{2}, \ldots, \xi_{N}\right]^{T}$, it is not difficult to verify that the above function is convex. In the following, we commence with the assumption that $\boldsymbol{\xi}=\mathbf{1}$, i.e., $\xi_{n}=1, \forall n \in \mathcal{N}$. Accordingly, we define $P_{c}^{\prime}(s)$ as follows:

$$
P_{c}^{\prime}(s)=\sum_{n=1}^{N}\left(-s_{n} \ln F_{n}+s_{n} \ln s_{n}\right) .
$$

By considering the constraint $\sum_{n=1}^{N} \tau_{n}^{*}\left(s_{n}\right) \leq 1$, the optimization problem in (6) can be expressed as follows:

$$
\min P_{c}^{\prime}(s)
$$




$$
\text { s.t. } \prod_{n=1}^{N} s_{n} \geq K
$$

where $K=e^{-\pi \lambda r^{2}} \prod_{n=1}^{N} F_{n}$.

1) Elimination of the Constraints: The constrained optimization problem in (13) involves a logarithmic function, and it requires great computational burden to be solved by using traditional sub-gradient method [16]. Therefore, we transform the above constrained optimization problem into a non-constrained optimization problem at first. In general, the exterior penalty function method can be applied to eliminate constraints in convex optimization problems [17]. However, the solution obtained by solving the transformed non-constrained optimization problem may not satisfy the constraints given before. Meanwhile, as the penalty factor approaches to infinity, the corresponding Hessian matrix becomes infinite which means that the computational complexity grows rapidly. Therefore, we intend to apply the multiplier penalty function method to transform the constrained optimization problem in (13) into an unconstrained one, which can eliminate the constraints without calculating infinite penalty factor.

We first introduce the variable $y$ to transform the inequality constraint into the equality constraint. The optimization problem in (13) can be rewritten as follows:

$$
\begin{gathered}
\min P_{c}^{\prime}(s), \\
\text { s.t. } \\
\left(\prod_{n=1}^{N} s_{n}-K\right)-y^{2}=0 .
\end{gathered}
$$

Then, we can obtain the corresponding augmented Lagrangian function as follows:

$$
\begin{aligned}
& \tilde{\phi}(s, w, \sigma, y) \\
& =\sum_{n=1}^{N}\left(-s_{n} \ln F_{n}+s_{n} \ln s_{n}\right) \\
& -w\left(\prod_{n=1}^{N} s_{n}-K-y^{2}\right)+\frac{\sigma}{2}\left(\prod_{n=1}^{N} s_{n}-K-y^{2}\right)^{2},
\end{aligned}
$$

where $w$ is the Lagrange multiplier, $\sigma$ is the penalty factor. Accordingly, the optimization problem in (13) can be converted to the following equivalent problem:

$$
\min \tilde{\phi}(s, w, \sigma, y) .
$$

Regarding $y$ as the only variable in above problem (16), we can achieve the minimum by applying the method of completing the square. Then, $y$ can be calculated as follows:

$$
\begin{aligned}
& y= \\
& \begin{cases}\left(\prod_{n=1}^{N} s_{n}-K\right)-w / \sigma, & \sigma\left(\prod_{n=1}^{N} s_{n}-K\right)-w \geq 0, \\
0, & \sigma\left(\prod_{n=1}^{N} s_{n}-K\right)-w<0 .\end{cases}
\end{aligned}
$$

(15). Then, we have:

$$
\begin{aligned}
\phi(s, w, \sigma) & =\sum_{n=1}^{N}\left(-s_{n} \ln F_{n}+s_{n} \ln s_{n}\right) \\
& +\frac{1}{2 \sigma}\left\{\left[\left(w-\sigma \prod_{n=1}^{N} s_{n}+\sigma \mathrm{K}\right)^{+}\right]^{2}-w^{2}\right\} .
\end{aligned}
$$

Therefore, the equivalent non-constrained optimization problem can be expressed as follows:

$$
\min \phi(s, w, \sigma)
$$

where

$$
\begin{aligned}
\phi(s, w, \sigma) & =\left\{\begin{array}{c}
g_{1}(s, w, \sigma), \prod_{n=1}^{N} s_{n}-K>w / \sigma, \\
g_{2}(s, w, \sigma), \prod_{n=1}^{N} s_{n}-K \leq w / \sigma,
\end{array}\right. \\
g_{1}(s, w, \sigma) & =\sum_{n=1}^{N}\left(-s_{n} \ln F_{n}+s_{n} \ln s_{n}\right)-w^{2} / 2 \sigma, \\
g_{2}(s, w, \sigma) & =\sum_{n=1}^{N}\left(-s_{n} \ln F_{n}+s_{n} \ln s_{n}\right) \\
& +\frac{1}{2 \sigma}\left\{\left[w-\sigma \prod_{n=1}^{N} s_{n}+\sigma \mathrm{K}\right]^{2}-w^{2}\right\} .
\end{aligned}
$$

Correspondingly, we have the following theorem.

Theorem 2: The non-constrained optimization problem in (19) is equivalent to the optimization problem in (13).

Proof: In light of [18], the optimal solution of the nonconstrained problem in (19) is equivalent to the local optimal solution of the constrained problem in (13). According to the definition of $P_{c}^{\prime}$, we can readily establish that:

$$
\begin{gathered}
\frac{\partial^{2} P_{c}^{\prime}}{\left(\partial s_{n}\right)^{2}}=\frac{1}{s_{n}}>0, \\
\frac{\partial^{2} P_{c}^{\prime}}{\partial s_{n} \partial s_{m}}=0, \forall n \neq m .
\end{gathered}
$$

Therefore, the Hessian matrix of $P_{c}^{\prime}$ is positive definite and $P_{c}^{\prime}$ is strictly convex. Consequently, the local optimal solution of the constrained optimization problem in (13) is the global optimal solution. Therefore, the non-constrained optimization problem in (19) is equivalent to the constrained optimization problem in (13).

2) The Proposed Edge Caching Resource Pricing Algorithm: To achieve the optimal solution of the optimization problem in (19), we need to update the Lagrange multiplier $w$ iteratively to revise the augmented Lagrangian function. Let $w^{(t)}$ denote the Lagrange multiplier for the $t$ th iteration. Then, the iterative relationship between $w^{(t+1)}$ and $w^{(t)}$ can

Substitute (17) into the augmented Lagrangian function in 


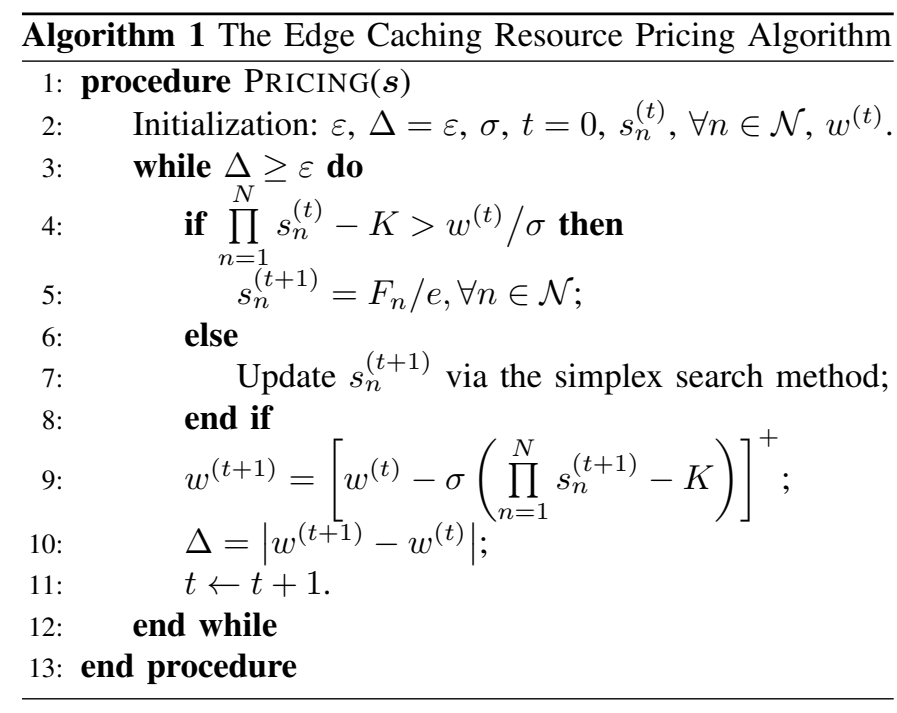

be established as follows:

$$
w^{(t+1)}=\left[w^{(t)}-\sigma\left(\prod_{n=1}^{N} s_{n}-K\right)\right]^{+} .
$$

After the Lagrange multiplier $w$ converges to its optimal point, the price vector $s$ becomes the only variable in both $g_{1}(\boldsymbol{s}, w, \sigma)$ and $g_{2}(\boldsymbol{s}, w, \sigma)$ since the penalty factor $\sigma$ is fixed.

$g_{1}(\boldsymbol{s}, w, \sigma)$ is a convex function over the variable $s_{n}$. Accordingly, the optimal solution can be achieved by taking the first derivative with respect to $s_{n}$. Let the first derivative equal to zero, $s_{n}$ can be obtained as follows:

$$
s_{n}=F_{n} / e, \forall n \in \mathcal{N},
$$

where $e$ is the base of a natural logarithm.

For $g_{2}(\boldsymbol{s}, w, \sigma)$, we propose to achieve the optimal solution by applying the simplex search method, which solves the non-constrained optimization problem without derivation. Therefore, this method has low computational complexity. The detailed description of the algorithm to achieve the optimal pricing strategy is shown in Algorithm 1.

\section{The Existence and Uniqueness of the NE}

NE offers a predictable and stable outcome. For the proposed Stackelberg game, we have the following theorem.

Theorem 3: There exists one and only one NE point in the Stackelberg game.

Proof: For the proposed Stackelberg game, we use the backward induction method to achieve the NE. Therefore, we should obtain the NE of the sub-game among followers at first. The sub-game is a non-cooperative game, formulating the competition among the CPs. To prove this theorem, we need to prove that there exists only one NE point for the noncooperative sub-game.

According to [19], we know that the NE exists if the players' strategy spaces are non-empty and closed-bounded sets in the Euclidean space. Note that the profit function $P_{n}$ for $\mathrm{CP} n$ is continuous with respect to $\tau_{n}$. In addition, the profit function
$P_{n}$ is a convex function. Therefore, it can be verified that each CP's strategy space is a non-empty and closed-bounded convex set in the Euclidean space. Correspondingly, the existence of the NE in the sub-game can be proved. It is obvious that the Hessian matrix of $P_{n}$ is negative definite. Accordingly, the profit function for each $\mathrm{CP}$ is strictly convex, and the optimization problem in (7) has a unique optimal solution. The uniqueness of the NE in the sub-game has been proven.

Since there is only one leader in the proposed Stackelberg game, and the leader's profit function is strictly convex as proven. It means that the optimal solution of the optimization problem in (6) is unique. Consequently, the existence and uniqueness of the NE in the proposed Stackelberg game has been proven.

\section{Simulation Results}

In this section, the performance of the proposed edge caching resource allocation strategy is evaluated via simulations. We consider a scenario including one cloud sever and four CPs, i.e., $N=4$. The average service radius $r$ of each F-AP is set to $0.5 \mathrm{~km}$. We set the preference parameter of contents for four CPs as $\beta_{1}=0.4, \beta_{2}=0.8, \beta_{3}=1.2$, and $\beta_{4}=1.6$. Each $\mathrm{CP}$ has the same number of contents, i.e., $C_{1}=C_{2}=\cdots=C_{N}=5000$, and these contents have same size. Accordingly, the number of content that each F-AP can cache for $N$ CPs can be set to $Q=Q_{1}=Q_{2}=\cdots=Q_{N}$.

Fig. 2 shows the leasing strategies for four CPs versus number of iterations. As shown, the proposed edge caching

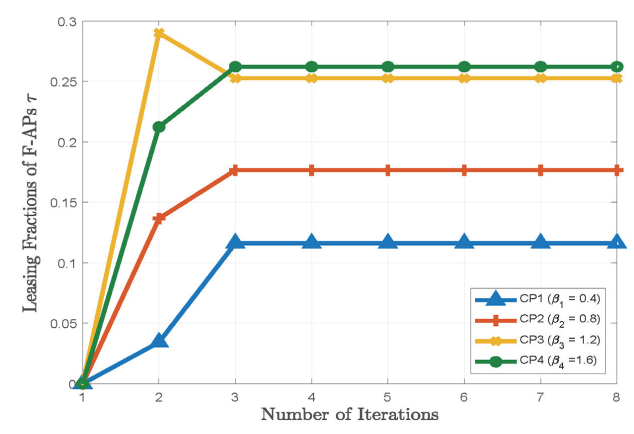

Fig. 2. Leasing fractions of F-APs for the CPs versus number of iterations.

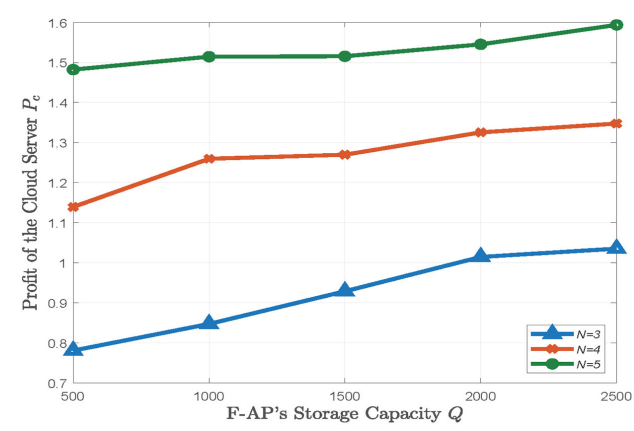

Fig. 3. The profit of the cloud server versus F-AP's storage capacity with different $N$. 


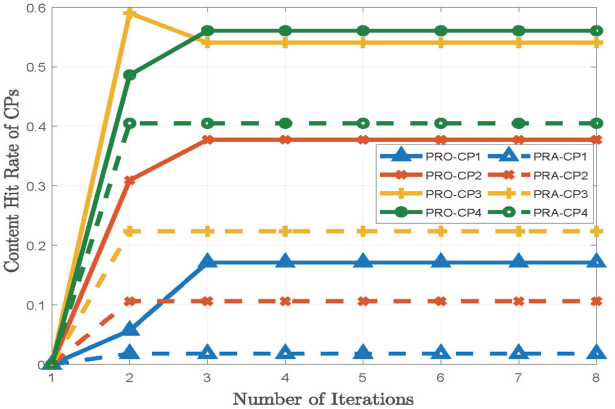

Fig. 4. The content hit rate versus number of iterations for different strategies.

resource allocation strategy can converge to a stable state quickly. It can be observed that the $\mathrm{CP}$ which has larger preference parameter of contents tends to rent more F-APs. Larger preference parameter of contents means the content popularity distribution is more concentrated. Therefore, for this kind of CPs, leasing F-APs to cache contents can bring higher content hit rate and then increase its profit.

Fig. 3 shows the profit of the cloud server versus F-AP's storage capacity $Q$ with different $N$. It can be observed that the cloud server's profit gradually increases as $Q$ ranges from 500 to 2500. The number of CPs can also influence the cloud server's profit. More participants bring the cloud server higher profit. The reason is that larger $Q$ and $N$ can make the competition among the CPs more intense and hence bring the cloud server more benefit.

In Fig. 4, we show the comparisons of the content hit rate between our proposed strategy (PRO) and the price-base resource allocation strategy (PRA) in [8] under the same scenario. It can be observed that the content hit rate of PRO is apparently superior to that of PRA. The reason is that the CPs in the latter strategy cache the contents with equal probability. However, in our proposed strategy, the CPs select the contents to cache with consideration of the content popularity distribution.

\section{CONCLUSIONS}

In this paper, we have proposed an edge caching resource allocation strategy in the F-RAN. Considering the content hit rate and leasing cost of the edge caching resource, we have formulated a Stackelberg game via the incentive mechanism. By exploiting the multiplier penalty function method and the simplex search method, we have obtained the optimal edge caching resource allocation strategy. Meanwhile, the existence and uniqueness of the NE in the proposed Stackelberg game have been proven. Simulation results have shown that significant improvement in terms of content hit rate is achieved by using our proposed strategy.

\section{ACKNOWLEDGEMENT}

This work was supported in part by the Natural Science Foundation of China under grant 61521061 and grant
61871122, the Natural Science Foundation of Jiangsu Province under grant BK20181264, the Research Fund of the State Key Laboratory of Integrated Services Networks (Xidian University) under grant ISN19-10, the Research Fund of the Key Laboratory of Wireless Sensor Network \& Communication (Shanghai Institute of Microsystem and Information Technology, Chinese Academy of Sciences) under grant 2017002, the National Basic Research Program of China (973 Program) under grant 2012CB316004, and the U.K. Engineering and Physical Sciences Research Council under grant EP/K040685/2.

\section{REFERENCES}

[1] M. Peng, S. Yan, K. Zhang, and et al., "Fog-computing-based radio access networks: Issues and challenges," IEEE Netwk., vol. 30, no. 4, pp. 46-53, Jul. 2016.

[2] Y. Jiang, M. Ma, M. Bennis, and et al., "User preference learning based edge caching for fog radio access network," IEEE Trans. Commun., vol. 67 , no. 2 , pp. 1268-1283, Feb. 2019.

[3] Y. Jiang, W. Huang, M. Bennis, and F. Zheng, "Decentralized asynchronous coded caching design and performance analysis in fog radio access networks," IEEE Trans. Mobile Computing (Early Access), pp. 1-12, Jan. 2019.

[4] T. D. Tran and L. B. Le, "Joint resource allocation and content caching in virtualized multi-cell wireless networks," IEEE Golbecom., pp. 1-6, Dec. 2017.

[5] J. C. A. Khreishah and A. Gharaibeh, "Joint caching, routing, and channel assignment for collaborative small-cell cellular networks," IEEE J. Sel. Areas Commun., vol. 34, no. 8, pp. 2275-2284, Aug. 2016.

[6] L. Shi, L. Zhao, G. Zheng, Z. Han, and Y. Ye, "Incentive design for cache-enabled D2D underlaid cellular networks using stackelberg game," IEEE Trans. Veh. Technol., vol. 68, no. 1, pp. 765-779, Jan. 2019.

[7] K. Zhao, S. Zhang, N. Zhang, and et al., "Incentive mechanism for cached-enabled small cell sharing: A stackelberg game approach," IEEE Golbecom., pp. 1-6, Dec. 2017.

[8] J. Li, H. Chen, Y. Chen, and et al., "Pricing and resource allocation via game theory for a small-cell video caching system," IEEE J. Sel. Areas Commun., vol. 34, no. 8, pp. 2115-2129, Aug. 2016.

[9] K. Shanmugam, N. Golrezaei, A. G. Dimakis, and et al., "Femto caching: Wireless content delivery through distributed caching helpers," IEEE Trans. Inf. Theory, vol. 59, no. 12, pp. 8402-8413, Dec. 2013.

[10] M. Haenggi, J. Andrews, F. Baccelli, and et al., "Stochastic geometry and random graphs for the analysis and design of wireless networks," IEEE J. Sel. Areas Commun., vol. 27, no. 7, pp. 1029-1046, Sep. 2009.

[11] S. H. Chae, T. Q. S. Quek, and W. Choi, "Content placement for wireless cooperative caching helpers: A tradeoff between cooperative gain and content diversity gain," IEEE Trans. Wireless Commun., vol. 16, no. 10, pp. 6795-6807, Oct. 2017.

[12] D. Fudenberg and J. Tirole, Game Theory. Cambrige, Massachusetts: The MIT Press, 1993.

[13] Y. Jiang, N. Lu, Y. Chen, F. Zheng, M. Bennis, X. Gao, and X. You, "Energy efficient non-cooperative power control in small cell networks," IEEE Trans. Veh. Technol., vol. 66, no. 8, pp. 7540-7547, Aug. 2017.

[14] X. Kang, R. Zhang, and M. Motani, "Price-based resource allocation for spectrun-sharing femtocell networks: A stackelberg game approach," IEEE J. Sel. Areas Commun., vol. 30, no. 3, pp. 538-549, Apr. 2012.

[15] H. Sun, F. Zhou, and R. Q. Hu, "Joint offloading and computation energy efficiency maximization in a mobile edge computing systems," IEEE Trans. Veh. Technol., vol. 68, no. 3, pp. 3052-3056, Mar. 2019.

[16] F. Zhou, Y. Wu, R. Q. Hu, and Y. Qian, "Computation rate maximization in UAV-enabled wireless powered mobile-edge computing systems," IEEE J. Sel. Areas Commun., vol. 36, no. 9, pp. 1927-1941, Sep. 2018.

[17] Y. Jiang, Q. Liu, F. Zheng, and et al, "Energy efficient joint resource allocation and power control for D2D communication," IEEE Trans. Veh. Technol., vol. 65, no. 8, pp. 6119-6127, Aug. 2016.

[18] M. J. D. Powell, Nonlinear Optimization. London: Academic Press, 1982.

[19] J. B. Rosen, "Existence and uniqueness of equilibrium points for concave n-person games," Econometrica, vol. 33, no. 3, pp. 520-534, Jul. 1965. 